Article

\title{
Decompositions of Weakly Compact Valued Integrable Multifunctions
}

\author{
Luisa Di Piazza ${ }^{1, *(1)}$ and Kazimierz Musiał ${ }^{2}$ (D) \\ 1 Department of Mathematics, University of Palermo, Via Archirafi 34, 90123 Palermo, Italy \\ 2 Institut of Mathematics, Wrocław University, Pl. Grunwaldzki 2/4, 50-384 Wrocław, Poland; \\ kazimierz.musial@math.uni.wroc.pl \\ * Correspondence: luisa.dipiazza@unipa.it
}

Received: 21 April 2020; Accepted: 21 May 2020; Published: 26 May 2020

check for updates

\begin{abstract}
We give a short overview on the decomposition property for integrable multifunctions, i.e., when an "integrable in a certain sense" multifunction can be represented as a sum of one of its integrable selections and a multifunction integrable in a narrower sense. The decomposition theorems are important tools of the theory of multivalued integration since they allow us to see an integrable multifunction as a translation of a multifunction with better properties. Consequently, they provide better characterization of integrable multifunctions under consideration. There is a large literature on it starting from the seminal paper of the authors in 2006, where the property was proved for Henstock integrable multifunctions taking compact convex values in a separable Banach space $X$. In this paper, we summarize the earlier results, we prove further results and present tables which show the state of art in this topic.
\end{abstract}

Keywords: gauge multivalued integral; scalarly defined multivalued integral; decomposition of a multifunction

MSC: 28B20; 26E25; 26A39; 28B05; 46G10; 54C60; 54C65

\section{Introduction}

Various investigations in mathematical economics, optimal control and multivalued image reconstruction led to study of the integrability of multifunctions. In fact, the multivalued integration has shown to be a useful tool when modeling theories in different fields [1-7]. Also, the study of multivalued integrals arises in a natural way in connection with statistical problems (see, for example, [8-10]). But the topic is interesting also from the point of view of measure and integration theory, as we can see in the papers $[1,7,11-38]$.

Here we examine two groups of the integrals: those functionally determined (we call them "scalarly defined integrals") (as Pettis, Henstock-Kurzweil-Pettis, Denjoy-Pettis integrals) and those identified by gauges (we call them "gauge defined integrals") as Henstock, McShane and Birkhoff integrals. The last class also includes versions of Henstock and McShane integrals, when only measurable gauges are allowed, and the variational Henstock and McShane integrals. We investigate only multifunctions with weakly compact and convex values. More general theory of integration is not sufficiently developped until now.

In particular, decomposition properties are considered both for scalarly defined integrals and for gauge defined integrals. The results presented here are contained in some papers quoted in the bibliography or can be easily obtained. Only some results are discussed. The novelty of the present article relies in the fact that we sumarize the results known until now in the field. Moreover, we compare them and in Table 2A,B we provide a clear view of the state of art in the topic. 


\section{Preliminaries}

Throughout the paper $X$ is a Banach space with norm $\|\cdot\|$ and its dual $X^{*}$. The closed unit ball of $X$ is denoted $B_{X}$. The symbol $c w k(X)$ denotes the collection of all nonempty convex weakly compact subsets of $X$. For every $C \in \operatorname{cwk}(X)$ the support function of $C$ is denoted by $s(\cdot, C)$ and defined on $X^{*}$ by $s\left(x^{*}, C\right)=\sup \left\{\left\langle x^{*}, x\right\rangle: x \in C\right\}$, for each $x^{*} \in X^{*}$. We set $\|C\|_{h}=d_{H}(C,\{0\}):=\sup \{\|x\|: x \in C\}$, where $d_{H}$ is the Hausdorff metric on the hyperspace $c w k(X)$. Let $([0,1], \lambda, \mathcal{L})$ be the unit interval equipped with Lebesgue measure $\lambda$ and Lebesgue measurable sets $\mathcal{L}$, while $\mathcal{I}$ is the collection of all closed subintervals of $[0,1] . L_{0}$ is the collection of all strongly measurable $X$-valued functions defined on $[0,1]$. Unless otherwise noted, all investigated multifunctions are defined on $[0,1]$ and take values in $\operatorname{cwk}(X)$. A function $f:[0,1] \rightarrow X$ is called a selection of a multifunction $\Gamma$ if $f(t) \in \Gamma(t)$, for almost every $t \in[0,1]$.

We recall that if $\Phi: \mathcal{L} \rightarrow Y$ is an additive vector measure with values in a normed space $Y$, then the variation of $\Phi$ is the extended non negative function $|\Phi|$ whose value on a set $E \in \mathcal{L}$ is given by $|\Phi|(E)=\sup _{\pi} \sum_{A \in \pi}\|\Phi(A)\|$, where the supremum is taken over all partitions $\pi$ of $E$ into a finite number of pairwise disjoint members of $\mathcal{L}$. If $|\Phi|<\infty$, then $\Phi$ is called a measure of finite variation. If $\Phi$ is defined only on $\mathcal{I}$, the finite partitions considered in the definition of variation are composed by intervals. In this case we will speak of finite interval variation and we will use the symbol $\widetilde{\Phi}$, namely:

$$
\widetilde{\Phi}([0,1])=\sup \left\{\sum_{i}\left\|\Phi\left(I_{i}\right)\right\|:\left\{I_{1}, \ldots, I_{n}\right\} \text { is a finite interval partition of }[0,1]\right\}
$$

If $\left\{I_{1}, \ldots, I_{p}\right\}$ is a partition in $[0,1]$ into intervals and $t_{j} \in[0,1], j=1, \ldots, p$, then $\left\{\left(I_{j}, t_{j}\right)\right\}_{j=1}^{p}$ is called an $\mathcal{I}$-partition. If $\delta$ is a gauge (that is positive function) on $[0,1]$ and $I_{j} \subset\left[t_{j}-\delta\left(t_{j}\right), t_{j}+\delta\left(t_{j}\right)\right], j=$ $1, \ldots, p, p \in \mathbb{N}$, then the $\mathcal{I}$-partition is called $\delta$-fine.

Moreover a usefull tool in our investigation is the notion of variational measure generated by an interval multimeasure. Given an interval multimeasure $\Phi: \mathcal{I} \rightarrow c w k(X)$, we call variational measure $V_{\Phi}: \mathcal{L} \rightarrow \mathbb{R}$ generated by $\Phi$, the measure whose value on a set $E \in \mathcal{L}$ is given by

$$
V_{\Phi}(E):=\inf _{\delta}\{\operatorname{Var}(\Phi, \delta, E): \delta \text { is a gauge on } E\},
$$

where

$\operatorname{Var}(\Phi, \delta, E)=\sup \left\{\sum_{j=1}^{p}\left\|\Phi\left(I_{j}\right)\right\|_{h}:\left\{\left(I_{j}, t_{j}\right)\right\}_{j=1}^{p}\right.$ is a $\delta$-fine $\mathcal{I}$-partition, with $\left.t_{j} \in I_{j} \cap E, j=1, \ldots, p\right\}$

Now we recall here briefly the definitions of the integrals involved in this article. A scalarly integrable multifunction $\Gamma:[0,1] \rightarrow c w k(X)$ is Pettis integrable $\left(P_{e}\right)$ in $c w k(X)$, if for every set $A \in \mathcal{L}$ there exists a set $M_{\Gamma}(A) \in \operatorname{cwk}(X)$ such that $s\left(x^{*}, M_{\Gamma}(A)\right)=\int_{A} s\left(x^{*}, \Gamma\right) d \lambda$ for every $x^{*} \in X^{*}$. We write it as $(P) \int_{A} \Gamma d \lambda$ or $M_{\Gamma}(A)$. A multifunction $\Gamma:[0,1] \rightarrow c w k(X)$ is called Bochner integrable if it is Bochner measurable (i.e., there exists a sequence of simple multifunctions $\Gamma_{n}:[0,1] \rightarrow \operatorname{cwk}(X)$ such that for almost all $t \in[0,1]$ one has $\left.\lim _{n} d_{H}\left(\Gamma_{n}(t), \Gamma(t)\right)=0\right)$ and integrably bounded. We will denote the family by $L_{1}$.

A multifunction $\Gamma:[0,1] \rightarrow c w k(X)$ is said to be McShane $(M S)$ (resp. Henstock $(\mathrm{H})$ ) integrable on $[0,1]$, if there exists $\Phi_{\Gamma}([0,1]) \in c w k(X)$ with the property that for every $\varepsilon>0$ there exists a gauge $\delta$ on $[0,1]$ such that for each $\delta$-fine $\mathcal{I}$-partition $\left\{\left(I_{1}, t_{1}\right), \ldots,\left(I_{p}, t_{p}\right)\right\}$ of $[0,1]$ (with $t_{i} \in I_{i}$ for all $i$ ), we have

$$
d_{H}\left(\Phi_{\Gamma}([0,1]), \sum_{i=1}^{p} \Gamma\left(t_{i}\right) \lambda\left(I_{i}\right)\right)<\varepsilon
$$

If the gauges above are taken to be measurable, then we speak of $\mathcal{H}$ (resp. Birkhoff)-integrability on $[0,1]$. If $I \in \mathcal{I}$, then $\Phi_{\Gamma}(I):=\Phi_{\Gamma \chi_{I}}[0,1]$. 
Finally if, instead of Formula (1), we have

$$
\sum_{i=1}^{p} d_{H}\left(\Phi_{\Gamma}\left(I_{i}\right), \Gamma\left(t_{i}\right) \lambda\left(I_{i}\right)\right)<\varepsilon,
$$

we speak about variational Henstock $(v H)$ (resp. McShane (vMS)) integrability on $[0,1]$.

The definition of variational Henstock (resp. McShane) integral comes from the classical Saks-Henstock Lemma for real valued functions. In case of Banach valued functions, they coincide with the definitions of Henstock (resp. McShane) integral if and only if the Banach space is of finite dimension. In the other cases, the variational integrals possesse better properties than Henstock or McShane integrals. In particular, the notion of variational Henstock integrability is a usefull tool to study the diferrentiability of Pettis integrals (cf. [13] (Corollary 4.1)). Formula (2) is the natural extension of such integrals to the multivalued case.

Moreover by [18] (Theorem 6.6) $v H$-integrability and $v \mathcal{H}$ integrability coincide. In all the cases $\Phi_{\Gamma}$ : $\mathcal{I} \rightarrow c w k(X)$ is an additive interval multimeasure. A multifunction $\Gamma:[0,1] \rightarrow c w k(X)$ is said to be Henstock-Kurzweil-Pettis (HKP) integrable in cwk(X) if it is scalarly Henstock-Kurzweil (HK)-integrable and for each $I \in \mathcal{I}$ there exists a set $N_{\Gamma}(I) \in \operatorname{cwk}(X)$ such that $s\left(x^{*}, N_{\Gamma}(I)\right)=(H K) \int_{I} s\left(x^{*}, \Gamma(t)\right) d t$ for every $x^{*} \in X^{*}$. If an HKP-integrable $\Gamma$ is scalarly integrable, then it is called weakly McShane integrable (wMS). We recall that a function $f:[0,1] \rightarrow \mathbb{R}$ is Denjoy-Khintchine (DK) integrable ([39] (Definition 11)), if there exists an ACG function (cf. [39]) F such that its approximate derivative is almost everywhere equal to $f$.

A multifunction $\Gamma:[0,1] \rightarrow c w k(X)$ is Denjoy-Khintchine-Pettis $(D K P)$ integrable in cwk $(X)$, if for each $x^{*} \in X^{*}$ the function $s\left(x^{*}, \Gamma(\cdot)\right)$ is Denjoy-Khintchine integrable and for every $I \in \mathcal{I}$ there exists $C_{I} \in \operatorname{cwk}(X)$ with $(D K) \int_{I} s\left(x^{*}, \Gamma(t)\right) d t=s\left(x^{*}, C_{I}\right)$, for every $x^{*} \in X^{*}$.

A multifunction $\Gamma:[0,1] \rightarrow \operatorname{cwk}(X)$ satisfies the $D b$-condition (resp. DL-condition) if

$$
\text { supess }_{t} \operatorname{diam}(\Gamma(t))<\infty \quad\left(\operatorname{resp} . \bar{\int}_{0}^{1} \operatorname{diam}(\Gamma(t)) d t<+\infty \text {, where } \bar{\int}\right. \text { denotes the upper integral). }
$$

We say that a multifunction $\Gamma:[0,1] \rightarrow \operatorname{cwk}(X)$ is positive if $s\left(x^{*}, \Gamma(\cdot)\right) \geq 0$ a.e. for each $x^{*} \in X^{*}$ separately. Of course, if $0 \in \Gamma(t)$ for almost every $t \in[0,1]$, then $\Gamma$ is positive. As regards other definitions of measurability and integrability that are treated here and are not explained and the known relations among them, we refer to [3,15-20,26,36,38,40-42], in order not to burden the presentation.

\section{Intersections}

In this section we are going to highlight some relations among gauge integrability and functionally defined integrability for multifunctions in order to understand better the examples given before. Since we have inclusions

$$
D P \supset H K P \supset w M S \supset P e \text { and } D P \supset H K P \supset H \supset \mathcal{H} \supset v H=v \mathcal{H}
$$

only the pairs of different types of integrals are interesting. For what concernes the symbol subscript $f v$ it means that the corresponding integral is of finite variation.

In Table 1 Henstock, $\mathcal{H}$ and $v \mathcal{H}$-integrable functions possessing integrals of finite variation, are not taken into consideration. The reason is simple. In [21] (Theorem 4.5) it is proven that such multifunctions are McShane and Birkhoff integrable, respectively. For a similar reason $w M S$-integrable multifunctions with integrals of finite variation are omitted. $\Phi$ is the indefinite integral of $G$. 
Table 1. Intersections. Arbitrary G.

\begin{tabular}{|c|c|c|c|}
\hline$G G$ & $v H$ & $\mathcal{H}$ & $H$ \\
\hline$P_{e f v}$ & $L_{1}$ Remark 1 & $B i_{f v} \quad[18]$ (Theorem 4.3) & $M S_{f v}$ [18] (Theorem 3.3) \\
\hline$P_{e}$ & $P_{e} \cap L_{0}+V_{\Phi} \ll \lambda \quad$ Remark 1 & Bi [18] (Theorem 4.3) & MS [18] (Theorem 3.3) \\
\hline$w M S$ & $P_{e} \cap L_{0}+V_{\Phi} \ll \lambda$ if $c_{0} \nsubseteq \subseteq X$ Remark 1 & $B i$ if $c_{0} \nsubseteq \subseteq X$ Remark 1 & $M S$ if $c_{0} \nsubseteq \nsubseteq X$ Remark 1 \\
\hline
\end{tabular}

Remark 1. Observe that, using the Rådström embedding: $i: c w k(X) \rightarrow l_{\infty}\left(B_{X^{*}}\right)$ (see for example [43] or [19]) given by $i(A):=s(\cdot, A)$, we have that:

1. directly from the definitions and the Rådström embedding, a multifunction $G:[0,1] \rightarrow c w k(X)$ is Birkhoff (resp. Henstock, McShane, variationally Henstock) integrable if and only if $i \circ G$ is integrable in the same sense. For the Pettis integrability this is not true. However, for Bochner measurable multifunctions, we have that since $\{G(E): E \in \mathcal{L}\}$ is separable for the Hausdorff distance and then $G$ is Pettis integrable if and only if $i \circ G$ is Pettis integrable ([26] (Proposition 4.5)), so we have $P_{e}=M S=B i$ ( for strongly measurable vector valued functions, Pettis, McShane and Birkhoff integrability coincide (see [44] (Corollary 4C) and [45] (Theorem 10)).

2. $P_{e f v} \cap v H=L_{1}$ in Table 1 solves the problem of [46], where the authors noticed that $P_{e} \cap v H \neq L_{1}$ in case of functions. The inclusion $P_{e f v} \cap v H \supset L_{1}$ is clear. To prove the inclusion $P_{e f v} \cap v H \subset L_{1}$ take $G \in P_{e f v} \cap v H$. Then $i \circ G$ is strongly measurable ([17] (Proposition 2.8)) and vH-integrable.

If $M_{G}$ is the Pettis integral of $G$, then $i \circ M_{G}$ is a measure of finite variation and $i \circ M_{G}(I)=(v H) \int_{I} i \circ G$. It follows that $i \circ G$ is Pettis integrable and then Bochner integrable by [47] (Theorem 4.1) or [48] (Lemma 2). Now we may apply [17] (Proposition 3.6) to obtain variational McShane integrability of G.

3. The results for the $P_{e}$ row and $v H$ column follow from Remark 1, by [17] (Theorem 4.3, d) $\Leftrightarrow e$ )) and [13] (Corollary 4.1), since $G$ is $v H$ integrable if and only if the variational measure $V_{\Phi}$ of its multivalued Pettis integral $\Phi$ is $\lambda$-continuous ([19] (Theorem 3.3)). Example 1 shows what can happen in the $P_{e} \backslash v H$ case.

4. The results given in wMS row follow from the $P_{e}$ row and [49] (Theorem 18) or [50] (Theorem 4.4).

Example 1. There exists a Pettis integrable multifunction $G:[0,1] \rightarrow c w k(X)$ such that $0 \in G(t)$ for every $t \in[0,1]$ and the variational measure associated to its Pettis integral $V_{M_{G}} \nless \lambda$.

Proof. Let $g:[0,1] \rightarrow X$ be a Pettis integrable function such that the variational measure associated to its Pettis integral $v_{v_{g}} \nless \lambda$, where $v_{g}(E)=(P) \int_{E} g d \lambda$, (for the existence see [13] (Corollary 4.2, Remark 4.3)), then we take $G(t):=\operatorname{conv}\{0, g(t)\}$. The multifunction $G$ is Pettis integrable and $V_{v_{g}}(E) \leq V_{M_{G}}(E)\left([17]\right.$ (Proposition 2.7)). It follows that $V_{M_{G}} \nless \lambda$.

\section{Decompositions}

The decomposition of a multifunction $\Gamma$ integrable in a certain sense into a sum of one of its integrable selections and a multifunction integrable in a narrower sense, relies essentially in the two facts:

(1) Existence of a selection of $\Gamma$ integrable in the same sense as $\Gamma$.

(2) A particular behaviour with respect to the integration of a positive multifunction.

In particular, regarding the results on the existence of selections we can observe that:

Proposition 1. Let $X$ be any Banach space and let $\Gamma:[0,1] \rightarrow c w k(X)$.

(i) If $\Gamma$ is Pettis (resp. HKP, wMS or DKP) integrable in cwk(X), then each scalarly measurable selection of $\Gamma$ is Pettis (resp. HKP, wMS or DK) integrable (see [26] (Corollary 2.3, Theorem 2.5) and [31] (Proposition 3, Remark 3))); 
(ii) if $\Gamma$ is Henstock (resp. McShane) integrable, then it possesses at least one Henstock (resp. McShane) integrable selection (see [33] (Theorem 3.1) or [30] (Theorem 2) in case of a separable X and compact valued $\Gamma$ );

(iii) if $\Gamma$ is $\mathcal{H}$ (resp. Birkhoff) integrable, then it possesses at least one $\mathcal{H}$ (resp. Birkhoff) integrable selection (see [17,30] (Theorem 3.4), [18] (Proposition 4.1));

(iv) if $\Gamma$ is $v H$ integrable, then there exists at least one $v H$ integrable selection (see [18] (Theorem 5.1)); if $\Gamma$ takes convex compact values and is Bochner integrable, then it possesses at least one Bochner integrable selection (see [17] (Theorem 3.9)).

While, for positive multifunctions, the following relations are known:

Proposition 2. Let $X$ be any Banach space and let $G:[0,1] \rightarrow c w k(X)$. Then

(i) If $G$ is Henstock integrable (resp. H-integrable) and positive, then it is also McShane (resp. Birkhoff) integrable on [0,1] (see [18] (Proposition 3.1));

(ii) If $G$ is variationally Henstock integrable and positive, then $G$ is Birkhoff integrable (see [17] (Proposition 4.1));

(iii) If G is HKP (resp. DKP) integrable and positive, then G is Pettis integrable (see [31] (Lemma 1)).

In general it is not possible to write $\Gamma=G+f$ with the meaning explained before. We present below a few examples.

Example 2. There exists a Pettis integrable multifunction $G:[0,1] \rightarrow \operatorname{cwk}(X)$ such that $0 \in G(t)$ for every $t \in[0,1]$, but $G$ is not McShane integrable.

Proof. Let $g:[0,1] \rightarrow X$ be Pettis but not McShane integrable and let $G(t):=\operatorname{conv}\{0, g(t)\}$ be the multifunction determined by $g$. Then $G$ is positive and Pettis integrable (see [20] (Proposition 2.3)). But according to [20] (Theorem 2.7) G is not McShane integrable.

Example 3. Any multifunction $G$ from Example 2 cannot be represented as $G=H+h$, where $H$ is $M c S h a n e$ integrable and $h$ is Pettis integrable.

Proof. If $h$ is a Pettis integrable selection of $G$, then there exists a measurable function $\alpha:[0,1] \rightarrow[0,1]$ such that $h(t)=\alpha(t) g(t)$, for every $t \in[0,1]$.

We have for $H(t):=G(t)-h(t)=\operatorname{conv}\{-\alpha(t) g(t),[1-\alpha(t)] g(t)\}$

$$
\begin{aligned}
s\left(x^{*}, H(t)\right) & =\sup _{0 \leq a \leq 1}\left\langle x^{*},-a \alpha(t) g(t)+(1-a)[1-\alpha(t)] g(t)\right\rangle=\sup _{0 \leq a \leq 1}\left\langle x^{*}, g(t)[1-a-\alpha(t)]\right\rangle \\
& =\left\langle x^{*}, g(t)[1-\alpha(t)]\right\rangle+\sup _{0 \leq a \leq 1}\left\langle x^{*},-a g(t)\right\rangle=\left\langle x^{*}, g(t)[1-\alpha(t)]\right\rangle-\inf _{0 \leq a \leq 1}\left\langle x^{*}, a g(t)\right\rangle \\
& =\left\langle x^{*}, g(t)[1-\alpha(t)]\right\rangle+\left\langle x^{*}, g(t)\right\rangle^{-}
\end{aligned}
$$

If $H$ would be McShane integrable then, the family

$$
\left\{\left\langle x^{*},[1-\alpha(\cdot)] g(\cdot)\right\rangle+\left\langle x^{*}, g(\cdot)\right\rangle^{-}:\left\|x^{*}\right\| \leq 1\right\}
$$

would be McShane equiintegrable. But in such a case $-H$ is also McShane integrable. Since

$$
\begin{aligned}
s\left(x^{*},-H(t)\right) & =\sup _{0 \leq a \leq 1}\left\langle x^{*}, a \alpha(t) g(t)+(1-a)[-1+\alpha(t)] g(t)\right\rangle \\
& =-\left\langle x^{*},[1-\alpha(t)] g(t)\right\rangle+\left\langle x^{*}, g(t)\right\rangle^{+}
\end{aligned}
$$

the family $\left\{\left\langle x^{*},[-1+\alpha(\cdot)] g(\cdot)\right\rangle+\left\langle x^{*}, g(\cdot)\right\rangle^{+}:\left\|x^{*}\right\| \leq 1\right\}$ would be also McShane equiintegrable. Substracting (3) and (4), we obtain McShane equiintegrability of the family 


$$
\left\{[1-2 \alpha(\cdot)]\left\langle x^{*}, g(\cdot)\right\rangle-\left\langle x^{*}, g(\cdot)\right\rangle:\left\|x^{*}\right\| \leq 1\right\}=\left\{-2 \alpha(\cdot)\left\langle x^{*}, g(\cdot)\right\rangle:\left\|x^{*}\right\| \leq 1\right\} .
$$

That means that if $H$ is McShane integrable, then also $h$ is McShane integrable. Consequently, $G$ is McShane integrable, contradicting our assumption.

Below, we make usage of multifunctions determined by functions, that is the multifunctions of the shape $G(t)=\operatorname{conv}\{0, g(t)\}$, where $g$ is a Banach space valued function. We refere to [20], for the relations of integrability between $g$ and $G$. At this stage we recall only that Henstock integrability of $g$, in general, does not imply Henstock integrability of $G$. In fact let $g$ be a Henstock but non McShane integrable function. If, by contradiction, $G$ is Henstock integrable then, by [18] (Proposition 3.1), G is McShane integrable and then, by [20] (Theorem 2.7), $g$ is McShane integrable. For the relations among different types of integrability for vector valued functions we refer also to [51].

Remark 2. There is now an obvious question: Let $\Gamma:[0,1] \rightarrow \operatorname{cwk}(X)$ be a variationally Henstock (Henstock, $\mathcal{H}$ ) integrable multifunction. Does there exist a variationally Henstock (Henstock, $\mathcal{H}$ ) integrable selection $f$ of $\Gamma$ such that the integral of $G:=\Gamma-f$ is of finite variation?

Unfortunately, in general, the answer is negative. The argument is similar to that applied in [51]. Assume that $X$ is separable and $g$ is the $X$-valued function constructed in [46] that is vH-integrable (and so it is strongly measurable by [52]) as well as Pettis but not Bochner integrable (see [46]). Let $\Gamma(t):=\operatorname{conv}\{0, g(t)\}$. Then, $\Gamma$ is vH-integrable (see [17] (Example 4.7)) but it is not Bochner integrable because it possesses at least one vH-integrable selection that is not Bochner integrable (see [17] (Theorem 3.7). Let now $f \in \mathcal{S}_{v H}(\Gamma)$ and consider the multifunction $G:=\Gamma-f$. Clearly $G$ is $v H$-integrable (hence also Henstock and $\mathcal{H}$-integrable) and $G(t)=\operatorname{conv}\{-f(t), g(t)-f(t)\}$ for all $t \in[0,1]$.

If the integral of $G$ were of finite variation, then $G$ would be Bochner integrable. In fact by Proposition 2, $G$ is Pettis integrable. Since $G$ is compact valued and $X$ is separable, an application of [25] (Proposition 3.5) gives that also $i(G)$ ( $i$ is the Rådström embedding) is Pettis integrable. Moreover, since G is Bochner measurable, $i(G)$ is strongly measurable. Now the finite variation of $i(G)$ yields Bochner integrability of $i(G)$. So since $G$ is Bochner measurable it becomes Bochner integrable (an equivalent proof can be deduced from Remark 1). Therefore, the selections $-f, g-f$ would be Bochner integrable since they are strongly measurable and dominated by $\|G\|_{h}$. But that would mean that $g$ is Bochner integrable, contrary to the assumption.

The multifunction $\Gamma$ is also an example of a strongly measurable and Birkhoff (McShane) integrable multifunction (see [17] (Theorem 4.3)) that cannot be decomposed into Birkhoff (McShane) integrable multifunction with integral of finite variation and a selection.

Example 4. There exists a McShane integrable multifunction $G:[0,1] \rightarrow$ cwk $(X)$ such that $0 \in G(t)$ for every $t \in[0,1]$, but $G$ is not Birkhoff integrable. Moreover, $G$ cannot be represented as $G=H+h$, where $H:[0,1] \rightarrow c w k(X)$ is Birkhoff integrable and $h:[0,1] \rightarrow X$ is McShane integrable. G may be chosen with its integral of finite variation.

Proof. We take in Example 2 a function $g$ that is McShane but not Birkhoff integrable and follow the same calculations. The second assertion can be proved as that in Example 3. If $g$ is bounded, then the variation of the McShane integral of $G$ is finite. Phillips' function is an example of such a function. As proved in [53] (Example 2.1) it is McShane integrable but not Birkhoff.

Example 5. Let $X=\ell_{2}[0,1]$ and let $\left\{e_{t}: t \in(0,1]\right.$ be its orthonormal basis. Let $G(t):=$ conv $\left\{0, e_{t}\right\}, t \in$ $(0,1]$. Then $G$ is Birkhoff integrable and bounded (cf. [20] (Example 2.11)). G cannot be represented as $G=H+h$, where $h$ is a Birkhoff integrable selection of $G$ and $H$ is Bochner integrable.

Proof. Suppose that such a representation exists: $G=H+h$. Then there exists a measurable function $\alpha:[0,1] \rightarrow[0,1]$ such that $h(t)=\alpha(t) e_{t}$ for all $t \in(0,1]$. We may assume that $\alpha$ is positive on a set of positive Lebesgue measure. Then, $H(t)=\operatorname{conv}\left\{\alpha(t) e_{t},(1-\alpha(t)) e_{t}\right\}$. Since $H$ is - by definition - 
Bochner measurable, there exists a set $K \subset[0,1]$ of full measure such that $\{H(t): t \in K\}$ is separable in $d_{H}$. But if $t \neq t^{\prime}$, then

$$
d_{H}\left(H(t), H\left(t^{\prime}\right)\right) \geq \max \left\{\alpha(t), \alpha\left(t^{\prime}\right)\right\} .
$$

Hence there is $\varepsilon>0$ such that $d_{H}\left(H(t), H\left(t^{\prime}\right)\right) \geq \varepsilon>0$ on a set of positive measure. However, that contradicts the separability.

Proposition 3. Let $G:[0,1] \rightarrow c w k(X)$ be McShane integrable (hence also Henstock) such that its integral $M_{G}: \mathcal{L} \rightarrow c w k(X)$ is of finite variation. If $G:=H+h$, where $h$ is a McShane integrable selection of $G$, then the variation of the multiiintegral $M_{H}$ of $H$ is finite. Moreover $H$ is Birkhoff and variationally Henstock integrable.

Proof. Let $G$ be McShane integrable and such that $\left|M_{G}\right|<\infty$ (in [53] (Example 2.1) there is an example of such a $G$ that is also not Birkhoff integrable). Let $v_{h}$ be the McShane integral of $h$. Since $h$ is a selection of $G$, we have $v_{h}(E) \in M_{G}(E)$ for every $E \in \mathcal{L}$. Consequently $\left|v_{h}\right|[0,1] \leq\left|M_{G}\right|[0,1]<\infty$ and then $\left|M_{H}\right|[0,1] \leq\left|M_{G}\right|[0,1]+\left|v_{h}\right|[0,1]<\infty$. Moeover by [19] (Corollary 3.7) we get that $H$ is Birkhoff and variationally Henstock integrable.

Now, to provide the reader with a quick overview of decomposition results which can be derived from Propositions 1 and 2 and from the articles quoted in the list of references, we have collected the results in Table 2A,B for gauge integrals and in Tables 3 and 4 for scalarly defines integrals.

In the left column of the subsequent tables there are multifunctions $G$ of different type. In the first row there are functions $f$ with the corresponding properties. In the intersection of a row $\alpha$ and a column $\beta$ one finds a class $V$ of multifunctions $\Gamma$ together with equality or an inclusion.

- The notation $=V$ means that each element of $V$ can be represented as $G+f$, where $f$ is a selection of $\Gamma$ belonging to the class $\beta$ and $G$ is a member of the class $\alpha$. And conversely, if $G \in \alpha$ and $f \in \beta$, then $G+f \in V$.

- The inclusion $\subset V$ means that if $G \in \alpha$ and $f \in \beta$, then $G+f \in V$. While $\subsetneq V$ means that if $G \in \alpha$ and $f \in \beta$, then $G+f \in V$ but there are elements $\Gamma$ of $V$ that cannot be represented as $\Gamma=G+f$, where $G \in \alpha$ and $f$ is a selection of $\Gamma$ belonging to $\beta$. Clearly, one has always $\Gamma=\Gamma+0$ but, if zero function is not a selection of $\Gamma$ then this is not what we are looking for.

- The inclusion $\supset V$ means that each element of $V$ can be represented as $G+f$, where $f$ is a selection of $\Gamma$ belonging to the class $\beta$ and $G$ is a member of the class $\alpha$. While $\supsetneq V$ means additionally that sometimes $G+f \notin V$ for properly chosen $G$ and $f$.

- Question tag indicates that we do not know something.

In Table 2A,B we describe decomposition into gauge integrable multifunction and function. Similarly as in case of Table 1 Henstock, $\mathcal{H}$ and $v \mathcal{H}$-integrable functions possessing integrals of finite variation, are not taken into consideration, because such functions are McShane and Birkhoff integrable, respectively ([21] (Theorem 4.5)).

In the tables that follow the most significant results will be highlighted by a box. 
Table 2. Part A: Decomposition : $\Gamma=G+f$, arbitrary gauge defined $G$ and $f$. Part B: Decomposition: $\Gamma=G+f$, arbitrary gauge defined $G$ and $f$.

\begin{tabular}{|c|c|c|c|c|}
\hline \multicolumn{5}{|c|}{$\mathbf{A}$} \\
\hline$G f$ & $L_{1}$ & $B i_{f v}$ & $B i$ & $M S_{f v}$ \\
\hline$L_{1}$ & $=L_{1}$ & $\begin{array}{c}\subsetneq B i_{f v} \text { Example 5, } \\
{[54] \text { (Proposition 4.7) }}\end{array}$ & $\begin{array}{c}\subsetneq B i \text { Example 5, } \\
\text { [54] (Proposition 4.7), [51] (Example 2) }\end{array}$ & $\begin{array}{c}\subsetneq M S_{f v} \text { Proposition 3, Example 5, } \\
\text { [54] (Proposition 4.7) }\end{array}$ \\
\hline$B i_{f v}$ & $\subset B i_{f v}$ & $=B i_{f v}$ & $\begin{array}{c}\subsetneq \text { CBi Example 5, } \\
\text { [54] (Proposition 4.7), [51] (Example 2) }\end{array}$ & $\begin{array}{c}M S_{f v} \text { Proposition 3, Example 5, } \\
\text { [54] (Proposition 4.7) }\end{array}$ \\
\hline$B i$ & $\subsetneq B i$ Remark 3 & $\subsetneq B i$ Remark 3 & $=B i$ & $\subsetneq M S$ Example 4 \\
\hline$B i \cap v H$ & $\begin{array}{c}\subset B i \cap v H \\
\neq B i \cap v H ?\end{array}$ & $\begin{array}{c}\subsetneq H \\
\text { Remark } 3\end{array}$ & $\begin{array}{l}\subset B i \\
\neq B i ?\end{array}$ & $\subsetneq M S$ Example 4 \\
\hline$M S_{f v}$ & $\subset M S_{f v}$ & $\begin{array}{l}\subset M S_{f v} \\
\neq M S_{f v} ?\end{array}$ & $\begin{array}{l}\subset M S \\
\neq M S ?\end{array}$ & $=M S_{f v}$ \\
\hline MS & $\subset M S$ Remark 3 & $\subset M S$ Remark 3 & $\begin{array}{l}\subset M S \\
\neq M S ?\end{array}$ & $\subset M S$ Remark 3 \\
\hline \multicolumn{5}{|c|}{ B } \\
\hline$G f$ & MS & $H$ & $\mathcal{H}$ & $v \boldsymbol{H}=\boldsymbol{v} \mathcal{H}$ \\
\hline$L_{1}$ & $\begin{array}{c}\subsetneq M S \text { Example 5 } \\
\text { [54] (Proposition 4.7) } \\
\text { [51] (Example 2) }\end{array}$ & $\begin{array}{c}\subsetneq \mathrm{H} \text { Example } 5 \\
\text { [54] (Proposition 4.7) }\end{array}$ & $\begin{array}{c}\subsetneq \mathcal{H} \text { Example 5 } \\
{[54] \text { (Proposition 4.7) }}\end{array}$ & $\begin{array}{c}\subsetneq v H \\
{[18](\text { Remark 5.4) }}\end{array}$ \\
\hline$B i_{f v}$ & $\begin{array}{c}\subsetneq \subsetneq M S \text { Example 5, } \\
\text { [54] (Proposition 4.7), [51] (Example 2) }\end{array}$ & $\subsetneq H$ Remark 2 & $\subsetneq H$ Remark 2 & $\subsetneq H$ Remark 2 \\
\hline$B i$ & $\subsetneq$ MS Example 4 & $\subsetneq H$ Remark 3 & $\begin{array}{c}=\mathcal{H} \\
{[18](\text { Theorem 4.2) }}\end{array}$ & $\begin{array}{l}\subsetneq \mathcal{H} \& \supset v H \\
{[19] \text { (Cor. 3.7) }}\end{array}$ \\
\hline$B i \cap v H$ & $\subsetneq$ MS Proposition 3 & $\subsetneq \mathrm{H} \quad$ Remark 3 & $\subsetneq \mathcal{H} \quad$ Remark 3 & $\begin{array}{c}=v \boldsymbol{H} \\
\text { [18] (Theorem 5.3), } \\
\text { [19] (Cor. 3.7) }\end{array}$ \\
\hline$M S_{f v}$ & $\subsetneq M S, \quad$ [51] (Example 2) & $\subsetneq H$ Remark 2 & $\subsetneq H$ Remark 2 & $\subsetneq H \quad$ Remark 2 \\
\hline MS & $=M S$ & $=\boldsymbol{H}[18]$ (Theorem 3.2) & $\supsetneq \mathcal{H}$ [18] (Theorem 4.2) & $\subset \mathrm{H}$ \\
\hline
\end{tabular}


Table 3. $\Gamma=G+f$. $G$ and $f$ scalarly defined.

\begin{tabular}{|c|c|c|c|c|c|}
\hline$G f$ & $P_{e}$ & $P_{e f v}$ & $w M S$ & НКР & $D P$ \\
\hline$P_{e}$ & $=P_{e}$ & $\begin{array}{c}\subsetneq \mathrm{Pe} \\
\text { Remark } 3\end{array}$ & $=w M S$ & $\begin{array}{l}=\text { HKP, }[31] \text { (Theorem 1) } \\
{[51] \text { (Theorem 1) (sep. case) }}\end{array}$ & $\begin{array}{c}=\mathrm{DP} \\
\text { [21] (Theorem 3.5) }\end{array}$ \\
\hline$P_{e f v}$ & $\subset P_{e}$ & $=P_{e f v}$ & $\subset w M S$ & $\subset \mathrm{HKP}$ & $\subset \mathrm{DP}$ \\
\hline$w M S$ & $\begin{array}{c}\subsetneq w \text { w } \\
\text { [21] (Theorem 3.7) }\end{array}$ & $\begin{array}{c}\subsetneq f w M S \\
\text { [21] (Theorem 3.7) }\end{array}$ & $=w M S$ & $\begin{array}{c}=\text { HKP } \\
{[21] \text { (Theorem 3.5) }}\end{array}$ & $\begin{array}{c}=\mathrm{DP} \\
\text { [21] (Theorem 3.5) }\end{array}$ \\
\hline НКР & $\begin{array}{c}\subsetneq f H K P \\
{[21](\text { Theorem 3.7) }}\end{array}$ & $\begin{array}{c}\subsetneq \text { ᄃHKP } \\
\text { [21] (Theorem 3.7) }\end{array}$ & $\begin{array}{c}\subsetneq \subsetneq H K P \\
\text { [21] (Theorem 3.7) }\end{array}$ & $=\mathrm{HKP}$ & $\begin{array}{c}=\mathrm{DP} \\
{[21] \text { (Theorem 3.5) }}\end{array}$ \\
\hline$D P$ & $\begin{array}{c}\subsetneq D P \\
{[21] \text { (Theorem 3.7) }}\end{array}$ & $\begin{array}{c}\subsetneq D P \\
{[21] \text { (Theorem 3.7) }}\end{array}$ & $\begin{array}{c}\subsetneq D P \\
{[21] \text { (Theorem 3.7) }}\end{array}$ & $\begin{array}{c}\subsetneq D D P \\
{[21] \text { (Theorem 3.7) }}\end{array}$ & $=\mathrm{DP}$ \\
\hline
\end{tabular}


Table 4. $\Gamma=G+f$. Arbitrary $G$ and $f$.

\begin{tabular}{lcccr}
\hline$G f$ & $\boldsymbol{P e}$ & $w \boldsymbol{~}$ & $\boldsymbol{H K P}$ & $\boldsymbol{D P}$ \\
\hline $\mathbf{P e}+\mathbf{D L}$ & $=P_{e}+\mathrm{DL}$ & $=w M S+\mathrm{DL}$ & $=\mathrm{HKP}+\mathrm{DL}$ & $=\mathrm{DP}+\mathrm{DL}$ \\
\hline $\mathbf{P e}+\mathbf{D b}$ & $=P_{e}+\mathrm{Db}$ & $=w M S+\mathrm{Db}$ & $=\mathrm{HKP}+\mathrm{Db}$ & $=\mathrm{DP}+\mathrm{Db}$ \\
\hline
\end{tabular}

Remark 3. We observe that

1. (Bi,H)-cell and $(B i \cap v H, H)$-cell: Multifunction $\Gamma$ that is Henstock integrable but not $\mathcal{H}$-integrable cannot be decomposed as $\Gamma=G+f$ with Birkhoff integrable $G$. G is only McShane integrable.

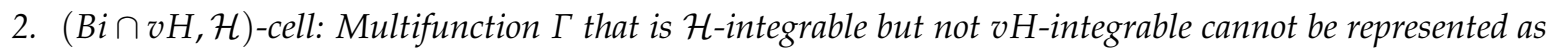
$\Gamma=G+f$ with Birkhoff and $v H$-integrable $G$.

3. The Henstock (resp. $\mathcal{H}$ ) integrability of $G$, together with $0 \in G(t)$ a.e. implies that $G$ is McShane integrable (resp. Bi) by [18] (Proposition 3.1) and then the characterization any class of $\Gamma$ is contained in the MS and Bi rows.

4. The $v H$ integrability of $G$, together with $0 \in G(t)$ a.e. implies that $G$ is Birkhoff integrable by [17] (Theorem 4.1), in particular if the selection $f$ is $v H$-integrable then we have $v H \ni \Gamma=G+f$ by [18] (Theorem 5.3), or [19] (Cor. 3.7).

5. $\left(M S, M S_{v f}\right)$-cell: Let $f$ be McShane integrable with $\left|v_{f}\right|[0,1]=+\infty$. Define $\Gamma$ by $\Gamma(t)=$ conv $\{f(t) / 2, f(t)\}$. The multifunction $\Gamma$ is McShane integrable and the integral of each scalarly measurable selection of $\Gamma$ is of infinite variation.

6. $\left(B i, L_{1}\right)$ and $\left(B i, B i_{v f}\right)$-cells: The same as in (5) but with a Birkhoff integrable function.

Now we are going to describe decompositions into scalarly integrable multifunctions and functions. In Table 3 there are no multifunctions that are $w M S, H K P$ or DP integrable and their integrals are of finite variation. In virtue of [54] (Theorem 3.2) such multifunctions are Pettis integrable.

If we assume in addition that $G$ satisfies the $D b$-condition (resp. DL-condition) we are able to find the relations below (cfr. [54] (Theorem 4.1)).

Remark 4. It seems that the decomposition $\Gamma=G+f$ with $G \in w M S \cup H K P \cup D P$ is useless if $\Gamma$ is Pettis or stronger integrable. If $\Gamma$ Henstock, $\mathcal{H}$ or $v H$ integrable, then Table $2 A, B$ give better decompositions. As an example in Table 5 we assume Pettis integrability of $G$.

Table 5. Decomposition: $\Gamma=G+f$, scalarly def. $G$ and gauge def. $f$

\begin{tabular}{lcccccccc}
\hline$G \boldsymbol{f}$ & $\boldsymbol{L}_{\mathbf{1}}$ & $\boldsymbol{B \boldsymbol { i } _ { f v }}$ & $\boldsymbol{B i}$ & $\boldsymbol{M} S_{f v}$ & $\boldsymbol{M S}$ & $\boldsymbol{H}$ & $\mathcal{H}$ & $\boldsymbol{v} \boldsymbol{H}=\boldsymbol{v \mathcal { H }}$ \\
\hline $\boldsymbol{P}_{\boldsymbol{e}}$ & $\supsetneq L_{1}$ & $\supsetneq B i_{f v}$ & $\supsetneq B i$ & $\supsetneq M S_{f v}$ & $\supsetneq M S$ & $\supsetneq M S$ & $\supsetneq B i$ & $\supsetneq v H$ \\
\hline $\boldsymbol{P}_{\boldsymbol{e} f v}$ & $\supsetneq L_{1}$ & $\supsetneq P e_{f v}$ & $\supsetneq B i_{f v}$ & $\supsetneq M S_{f v}$ & $\supsetneq M S_{f v}$ & $\supsetneq M S_{f v}$ & $\supsetneq B i_{f v}$ & $\supsetneq L_{1}$ \\
\hline
\end{tabular}

Remark 5. One would like to have yet decompositions $\Gamma=G+f$ with gauge defined $G$ and scalarly defined $f$. Unfortunately, the Pettis row in Table 3 seems to be top of what can be obtained. Pettis integrability seems to be resistant to gauge integrable selections. If $f$ is a Henstock integrable selection of a Pettis integrable $\Gamma:[0,1] \rightarrow c w k(X)$, then $\Gamma=G+f$ and $f$ is Pettis integrable. Hence $f$ is McShane integrable and $G$ is Pettis integrable. We are unable to conclude any stronger type integrability for $G$ (see Examples 2 and 3). Therefore, we do not present the corresponding table.

One could expect that if we assume Bochner measurability of $G$ and strong measurability of $f$ in the above tables, then we should get more information. Unfortunately, the answer is negative. The only positive fact is the equality of Pettis, McShane and Birkhoff integrabilities for multifunctions and functions and Bochner integrability in case of integrals of finite variation. Other interrelations remain exactly the same as in the tables presented above. 
Moreover, we want to recall that results on decompositions were also obtained for scalarly defined and gauge integrals in the fuzzy setting, as generalization of the multivalued case, in the papers [55-57].

\section{Conclusions}

As we wrote in the introduction, a more general theory for the multivalued integration is not sufficiently developped until now. In the particular case of closed convex sets, only some results are known [21]. It should be interesting to also develop the theory in such a more general case.

Author Contributions: The authors contributed jointly to this work, have read and agreed to the published version of the manuscript. All authors have read and agreed to the published version of the manuscript.

Funding: This research was partially supported by Grant "Analisi reale, teoria della misura ed approssimazione per la ricostruzione di immagini" (2020) of GNAMPA-INDAM (Italy) and by University of Palermo-Fondo Ricerca di Base 2019).

Conflicts of Interest: The authors declare no conflict of interest.

\section{References}

1. Balder, E.J.; Sambucini, A.R. Fatou's lemma for multifunctions with unbounded values in a dual space. J. Convex Anal. 2005, 12, 383-395.

2. Cichon, K.; Cichon, M. Some Applications of Nonabsolute Integrals in the Theory of Differential Inclusions in Banach Spaces. In Vector Measures, Integration and Related Topics; Curbera, G.P., Mockenhaupt, G., Ricker, W.J., Eds.; Operator Theory: Advances and Applications; BirHauser-Verlag: Basel, Switzerland, 2010; Volume 201, pp. 115-124, ISBN 978-3-0346-0210-5.

3. Di Piazza, L.; Marraffa, V.; Satco, B. Set valued integrability and measurability in non separable Fréchet spaces and applications. Math. Slovaca 2016, 66, 1119-1138. [CrossRef]

4. Di Piazza, L.; Marraffa, V.; Satco, B. Closure properties for integral problems driven by regulated functions via convergence results. J. Math. Anal. Appl. 2018, 466, 690-710. [CrossRef]

5. Di Piazza, L.; Satco, B. A new result on impulsive differential equations involving non-absolutely convergent integrals. J. Math. Anal. Appl. 2009, 352, 954-963. [CrossRef]

6. Hu, S.; Papageorgiou, N.S. Handbook of Multivalued Analysis I and II. In Mathematics and Its Applications, 419; Kluwer Academic Publisher: Dordrecht, The Netherlands, 1997.

7. Labuschagne, C.C.A.; Marraffa, V. On spaces of Bochner and Pettis integrable functions and their set-valued counterparts. Nonlinear Math. Uncertainty Appl. AISC 2011, 100, 51-59.

8. Kudo, H. Dependent experiments and sufficient statistics. Nat. Sci. Rep. Ochanomizu Univ. 1954, 4, 151-163.

9. Richter, H. Verallgemeinerung eines in der Statistik benötigten Satzes der Masstheorie, (German). Math. Ann. 1963, 150, 85-90. [CrossRef]

10. Shang, Y. The limit behavior of a stochastic logistic model with individual time-dependent rates. J. Math. 2013, 2013, 1-8. [CrossRef]

11. Boccuto, A.; Candeloro, D.; Sambucini, A.R. Henstock multivalued integrability in Banach lattices with respect to pointwise non atomic measures. Atti Accad. Naz. Lincei Rend. Lincei Mat. Appl. 2015 26, 363-383. [CrossRef]

12. Boccuto, A.; Sambucini, A.R. A note on comparison between Birkhoff and McShane-type integrals for multifunctions. Real Anal. Exch. 2011, 37, 315-324. [CrossRef]

13. Bongiorno, B.; Di Piazza, L.; Musiał, K. A variational Henstock integral characterization of the Radon-Nikodym property. Ill. J. Math. 2009, 53, 87-99. [CrossRef]

14. Boxer, L. Multivalued Functions in Digital Topology. Note di Matematica 2017, 37, 61-76. [CrossRef]

15. Candeloro, D.; Croitoru, A.; Gavrilut, A.; Sambucini, A.R. An extension of the Birkhoff integrability for multifunctions. Mediterr. J. Math. 2016, 13, 2551-2575. [CrossRef]

16. Candeloro, D.; Croitoru, A.; Gavrilut, A.; Iosif, A.; Sambucini, A.R. Properties of the Riemann-Lebesgue integrability in the non-additive case. Rend. Circ. Mat. Palermo II Ser. 2019. [CrossRef]

17. Candeloro, D.; Di Piazza, L.; Musiał, K.; Sambucini, A.R. Gauge integrals and selections of weakly compact valued multifunctions. J. Math. Anal. Appl. 2016, 441, 293-308. [CrossRef] 
18. Candeloro, D.; Di Piazza, L.; Musiał, K.; Sambucini, A.R. Relations among gauge and Pettis integrals for multifunctions with weakly compact convex values. Annali di Matematica 2018, 197, 171-183. [CrossRef]

19. Candeloro, D.; Di Piazza, L.; Musiał, K.; Sambucini, A.R. Some new results on integration for multifunction. Ricerche Mat. 2018, 67, 361-372. [CrossRef]

20. Candeloro, D.; Di Piazza, L.; Musiał, K.; Sambucini, A.R. Multifunctions determined by integrable functions. Inter. J. Approx. Reason. 2019, 112, 140-148. [CrossRef]

21. Candeloro, D.; Di Piazza, L.; Musiał, K.; Sambucini, A.R. Integration of multifunctions with closed convex values in arbitrary Banach spaces. in press J. Convex Anal. 2020, 27.

22. Candeloro, D.; Sambucini, A.R. A Girsanov result through Birkhoff integral. In Computational Science and Its Applications ICCSA; Gervasi, O., Murgante, B., Misra, S., Stankova, E., Torre, C.M., Rocha, A.M.A.C., Taniar, D., Apduhan, B.O., Tarantino, E., Ryu, Y., Eds.; LNCS 10960; Springer: Cham, Switzerland, 2018; pp. 676-683.

23. Candeloro, D.; Sambucini, A.R.; Trastulli, L. A vector Girsanov result and its applications to conditional measures via the Birkhoff integrability. Mediterr. J. Math. 2019, 16, 144. [CrossRef]

24. Caponetti, D.; Marraffa, V.; Naralenkov, K. On the integration of Riemann-measurable vector-valued functions. Monatsh. Math. 2017, 182, 513-536. [CrossRef]

25. Cascales, C.; Kadets, V.; Rodríguez, J. Birkhoff integral for multi-valued functions. J. Math. Anal. Appl. 2004, 297, 540-560. [CrossRef]

26. Cascales, C.; Kadets, V.; Rodríguez, J. Measurable selectors and set-valued Pettis integral in non-separable Banach spaces. J. Funct. Anal. 2009, 256, 673-699. [CrossRef]

27. Cascales, C.; Kadets, V.; Rodríguez, J. The Gelfand integral for multi-valued functions. J. Convex Anal. 2011, 18, 873-895.

28. D'Aniello, E.; Mauriello, M. Some Types of Composition Operators on Some Spaces of Functions. arXiv 2020, arXiv:2005.07735.

29. Di Piazza, L.; Marraffa, V.; Musiał, K. Variational Henstock integrability of Banach space valued function. Math. Bohem. 2016, 141, 287-296. [CrossRef]

30. Di Piazza, L.; Musiał, K. A decomposition theorem for compact-valued Henstock integral. Monatsh. Math. 2006, 148, 119-126. [CrossRef]

31. Di Piazza, L.; Musiał, K. A decomposition of Denjoy-Khintchine-Pettis and Henstock-Kurzweil-Pettis integrable multifunctions. In Vector Measures, Integration and Related Topics; Curbera, G.P., Mockenhaupt, G., Ricker, W.J., Eds.; Operator Theory: Advances and Applications; Birkhauser Verlag: Basel, Switzerland, 2009; Volume 201, pp. 171-182.

32. Di Piazza, L.; Musiał, K. Henstock-Kurzweil-Pettis integrability of compact valued multifunctions with values in an arbitrary Banach space. J. Math. Anal. Appl. 2013, 408, 452-464. [CrossRef]

33. Di Piazza, L.; Musiał, K. Relations among Henstock, McShane and Pettis integrals for multifunctions with compact convex values. Monatsh. Math. 2014, 173, 459-470. [CrossRef]

34. Di Piazza, L.; Porcello, G. Radon-Nikodym theorems for finitely additive multimeasures. Z. Anal. Ihre. Anwend. (ZAA) 2015, 34, 373-389. [CrossRef]

35. Kaliaj, S.B. The New Extensions of the Henstock-Kurzweil and the McShane Integrals of Vector-Valued Functions. Mediterr. J. Math. 2018, 15, 22. [CrossRef]

36. Musiał, K. Pettis Integrability of Multifunctions with Values in Arbitrary Banach Spaces. J. Convex Anal. 2011, 18, 769-810.

37. Musiał, K. Approximation of Pettis integrable multifunctions with values in arbitrary Banach spaces. J. Convex Anal. 2013, 20, 833-870.

38. Naralenkov, K.M. A Lusin type measurability property for vector-valued functions. J. Math. Anal. Appl. 2014, 417, 293-307. [CrossRef]

39. Gordon, R.A. The Denjoy extension of the Bochner, Pettis and Dunford integrals. Stud. Math. 1989, 92, 73-91. [CrossRef]

40. Candeloro, D.; Sambucini, A.R. Order-type Henstock and McShane integrals in Banach lattices setting. In Proceedings of the Sisy 20014- IEEE 12th International Symposium on Intelligent Systems and Informatics, Subotica, Serbia, 11-13 September 2014; Volume 9.

41. El Amri K.; Hess, C. On the Pettis integral of closed valued multifunctions. Set-Valued Anal. 2000, 8, 329-360. [CrossRef] 
42. Shang, Y. Continuous-time average consensus under dynamically changing topologies and multiple time-varying delays. Appl. Math. Comput. 2014, 244, 457-466. [CrossRef]

43. Labuschagne, C.C.A.; Pinchuck, A.L.; van Alten, C.J. A vector lattice version of Rådström's embedding theorem. Quaest. Math. 2007, 30, 285-308. [CrossRef]

44. Fremlin, D.H. The generalized McShane integral. Ill. J. Math. 1995, 39, 39-67. [CrossRef]

45. Fremlin, D.H. The McShane and Birkhoff Integrals of Vector-Valued Functions; Mathematics Department Research Report 92-10, Version 18.5; University of Essex: Colchester, UK, 2007.

46. Di Piazza, L.; Marraffa, V. The McShane, PU and Henstock integrals of Banach valued functions. Czechoslov. Math. J. 2002, 52, 609-633. [CrossRef]

47. Musiał, K. Topics in the theory of Pettis integration. Rend. Istit. Mat. Univ. Trieste 1991, 23, 177-262.

48. Di Piazza, L.; Musiał, K. A characterization of variationally McShane integrable Banach-space valued function. Ill. J. Math. 2001, 45, 279-289. [CrossRef]

49. Gordon, R.A. The McShane integral of Banach-valued functions. Ill. J. Math. 1990, 34, 557-567.

50. Saadoune, M.; Sayyad, R. The weak Mc Shane integral. Czechoslov. Math. J. 2014, 64, 387-418. [CrossRef]

51. Di Piazza, L.; Musiał, K. Set-Valued Kurzweil-Henstock-Pettis Integral. Set-Valued Anal. 2005, 13, $167-179$. [CrossRef]

52. Cao, S. The Henstock integral for Banach-valued functions, The Henstock integral for Banach-valued functions. SEA Bull. Math. 1992, 16, 35-40.

53. Rodríguez, J. Some examples in Vector Integration. Bull. Aust. Math. Soc. 2009, 80, 384-392. [CrossRef]

54. Candeloro, D.; Di Piazza, L.; Musiał, K.; Sambucini, A.R. Multi-integrals of finite variation. Boll. dell'Unione Matematica Italiana 2020. [CrossRef]

55. Bongiorno, B.; Di Piazza, L.; Musiał, K. A Decomposition Theorem for the Fuzzy Henstock Integral. Fuzzy Sets Syst. 2012, 200, 36-47. [CrossRef]

56. Di Piazza, L.; Marraffa, V. Pettis integrability of fuzzy mappings with values in arbitrary Banach spaces, functions. Math. Slovaca 2017, 67, 1359-1370. [CrossRef]

57. Musiał, K. A decomposition theorem for Banach space valued fuzzy Henstock integral. Fuzzy Sets Syst. 2015, 259, 21-28. [CrossRef]

(C) 2020 by the authors. Licensee MDPI, Basel, Switzerland. This article is an open access article distributed under the terms and conditions of the Creative Commons Attribution (CC BY) license (http://creativecommons.org/licenses/by/4.0/). 